\title{
Management of metastatic tumors of the spine: strategies and operative indications
}

\author{
Michael P. Steinmetz, M.D., Anis Mekhail, M.D., and Edward C. Benzel, M.D. \\ Department of Neurosurgery, Cleveland Clinic Foundation, Cleveland, Ohio; and Department of \\ Orthopaedic Surgery, University of Illinois at Chicago, Illinois
}

\begin{abstract}
The spinal column is the most frequent site of bone metastasis in the body. Spine surgeons are often involved in the care of these patients only after nonoperative management has failed. Because surgery has been viewed as no better than radiotherapy in the treatment of metastasis of the spine, it has only been used as a salvage approach. These views are based on a body of literature in which laminectomy combined with radiotherapy was compared with radiotherapy alone. Anterior approaches to the spine are now popular and familiar to most surgeons. These approaches allow direct access to the metastatic lesion, reconstruction of the anterior vertebral column, and the placement of anterior instrumentation. Outcomes are frequently much better when this combined treatment is used instead of radiotherapy alone. In selected patients, surgery may be desired as first-line therapy before radio- or chemotherapy has been initiated.

The controversy surrounding surgery for metastatic spinal disease is reviewed. Treatment strategies, both operative and nonoperative, are presented. Indications and strategies for surgery are also presented, and the supporting literature is reviewed.
\end{abstract}

KEY WoRDS • metastasis • spine • operative indications

In North America approximately 18,000 new cases of spinal metastatic disease are diagnosed each year. ${ }^{32}$ Spine surgeons are often asked to evaluate these patients for the treatment of intractable pain or spinal cord compression caused directly or indirectly by the metastatic lesion(s). Medical care of these patients is expensive, and costs may be increased if the tumor produces paresis or paraplegia. ${ }^{15}$

The spinal column is the most frequent site of bone metastasis. ${ }^{19}$ Although the lumbar spine is more frequently involved with metastasis overall, in those patients who are symptomatic, the thoracic spine is involved in $70 \%$ of cases whereas the cervical and lumbar regions are affected in 10 and $20 \%$ of cases, respectively. ${ }^{15}$ Breast, lung, prostate, and renal carcinoma are the most common tumors that disseminate to the spine. ${ }^{6}$ Other tumors that commonly metastasize to the spine include myeloma, lymphoma, and gastrointestinal carcinoma.

Many believe that surgery combined with radiotherapy is no better than radiotherapy alone for the management of metastatic spinal disease. This contention is based on literature in which investigators have compared the effects of laminectomy and radiotherapy with those of radiotherapy alone. ${ }^{5,14}$ With advances in anterior spine surgery and instrumentation, operative intervention has proven to be very effective, perhaps better than radiation therapy in certain circumstances.

Abbreviations used in this paper: LECA $=$ lateral extracavitary approach; MMA = methylmethacrylate; $\mathrm{VB}=$ vertebral body.

\section{MANAGEMENT CONTROVERSY}

The dogma that surgery yields no better results than does nonoperative management has resulted in the majority of patients with spinal metastases being treated with a nonoperative strategy. Radiotherapy is the usual modality. In reports in which the authors review the older literature, no significant difference in neurological outcome was demonstrated between those treated with laminectomy/radiotherapy compared with those treated with radiotherapy alone. ${ }^{5,14}$ In these studies, surgery consisted of decompressive laminectomy alone. Simple laminectomy fails to address key issues paramount to the management of spinal metastasis. First, the lesion is most often located anteriorly in the VB, ${ }^{25}$ and laminectomy often fails to decompress fully the spinal cord from this anterior mass. Second, performing a laminectomy does not allow significant resection of an anteriorly located tumor. Of note, in these studies, spinal stability was not mentioned in its relationship to patient outcome. In a review published by Findlay ${ }^{12}$ no significant difference was found between laminectomy combined with radiotherapy and radiotherapy alone; however, postoperative neurological deterioration correlated with spinal instability. Based on this experience, physicians have often favored offering surgery only in selected cases. $^{7,26}$

Recently, anterior, antero-, and posterolateral approaches to the spine have become popular routes by which to reach anterior lesions for the reconstruction of the weightbearing anterior column and the placement of anterior instrumentation. Advances have been made in the under- 
standing of tumor biology and behavior. ${ }^{23}$ In light of our familiarity with anterior approaches, advances in spinal instrumentation, and increased understanding of the pathophysiology of spinal metastasis, some have argued that in selected cases surgery should be offered as first-line therapy as opposed to a salvage operation. ${ }^{13,15,29,31}$

\section{SURGICAL MANAGEMENT}

Surgery may be indicated for obtaining a diagnosis or for decompression and/or stabilization of the spine. The goals of surgery are to decrease pain, to preserve or to improve neurological function and to mobilize the patient without an external orthosis. With the refinement of computerized tomography-guided biopsy procedures, however, rarely is surgery required solely for diagnostic purposes. As previously mentioned, surgery had been judged to be no better than radiotherapy based on results obtained in studies of decompressive laminectomy. In fact, in selected patients, surgery may be indicated de novo prior to radiation therapy. ${ }^{29}$ Surgery prior to chemo- or radiotherapy probably decreases postoperative morbidity such as wound breakdown. In several studies, it has been documented that the use of prolonged steroid therapy and previous treatment with external-beam radiation therapy compromise wound healing and immune function, with a breakdown of the surgical wound in up to $30 \%$ of surgically treated patients. ${ }^{17,20,21,31}$ Certain considerations apply to specific tumor types. Preoperative embolization of metastatic renal cell carcinoma, for instance, significantly reduces intraoperative blood loss. ${ }^{25}$

Many surgical strategies may be followed in the treatment of metastatic spinal disease. Better outcomes appear to be demonstrated in those who have undergone an operation via the anterior-approach (that is, improved neurological status and length of survival). ${ }^{8,15,17,24,27-30}$ As noted previously, because most metastatic lesions occur in the $\mathrm{VB}$, an anterior approach offers the most direct route to the disease process. It also allows for minimum removal of uninvolved bone, rapid removal of tumor, effective reconstruction of the weight-bearing anterior column, short-segment fixation, and improved wound healing. ${ }^{15} \mathrm{In}$ some cases, patients are not candidates for an anterior approach, such as those who are at high medical risk or who harbor circumferential tumors. Posterior and posterolateral approaches may be more appropriate in these cases. Posterior-approach surgery has been condemned based on results published in old literature. In recent studies in which surgeons performed procedures such as transpe dicular and costotransversectomy approaches, the authors have reported comparable results to anterior-approach surgery, perhaps with a greater incidence of wound-related complications and cerebrospinal fluid fistulas. ${ }^{2,9}$ Combined anterior-posterior procedures are at times indicated. The combined approach allows circumferential tumor decompression and spinal stabilization, which may be indicated in cases in which there is significant instability or after anterior decompression has been performed at the cervicothoracic or thoracolumbar junction. The sacrifice for improved decompression and stability, however, is increased morbidity.

Contrary to surgery for trauma-induced or degenerative spine disease, the ultimate goal in metastatic disease is not fusion, but spinal stability achieved by placing instrumentation and struts. In this regard, foreign objects, such as titanium cages, are usually used as interbody struts, and often a bone graft is not placed. Spinal instrumentation and interbody struts will be required to confer spinal stability for the remainder of the patient's life, which is often shorter than the time required for a solid arthrodesis to develop. Thus, in cases of metastatic disease, rigid, fixed cantilever-beam instrumentation is often used. When possible, bicortical screw purchase of bone should be used. In the place of bone graft, MMA is often used because it sets solidly and provides immediate stability. It may be placed in the center of titanium cages or into a chest tube, as described by Errico and Cooper. ${ }^{11}$ They described placing a No. 36 French chest tube into a vertebrectomy site, in which the ends are fit into defects created in the VB above and below the area of resection. This tube is then filled with MMA (Fig. 1).

Instrumentation is often placed anteriorly if an anterior approach is performed, and posteriorly after a posterior approach. Constructs placed anteriorly are usually short segment, often placed one level above and one below the level of the disease process. Posteriorly, constructs are often placed three spinal levels above and two levels below the level of the lesion or longer if there is significant deformity present. When there is believed to be significant instability or major deformity, consideration should be given to the placement of anterior and supplemental posterior instrumentation. Often anterior-approach tumor resection at the cervicothoracic and thoracolumbar junctions should be augmented with posteriorly-placed instrumentation.

\section{REGIONAL NUANCES}

\section{Cervical Spine}

The subaxial spine may be approached either anteriorly though a standard incision along the sternocleidomastiod muscle or posteriorly via laminectomy. These approaches are familiar to most spine surgeons, and anterior or posterior instrumentation is readily applied in this region. Removal of the involved bone provides direct visualization of the spinal cord.

\section{Cervicothoracic Junction}

This region consists of the lower cervical vertebrae through T-1 and T-2. This region marks the junction of the mobile cervical spine and the relatively immobile thoracic spine. For resection of T-1 and T-2 lesions via the anterior approach, often a sternotomy or manubrial window will have to be created (Fig. 2). This should be performed from the patient's left side; the recurrent laryngeal nerve has a deeper course on this side and there is less chance of injury, although the thoracic duct is at risk during the leftsided approach. The great vessels may be retracted to allow visualization of T-1 to approximately T-3. The brachiocephalic vein may be ligated and incised, if needed, for increased visualization. ${ }^{26}$ Removal of involved bone allows visualization and decompression of the spinal cord. An interbody strut and anterior instrumentation may be placed through the same incision. The presence of tho- 


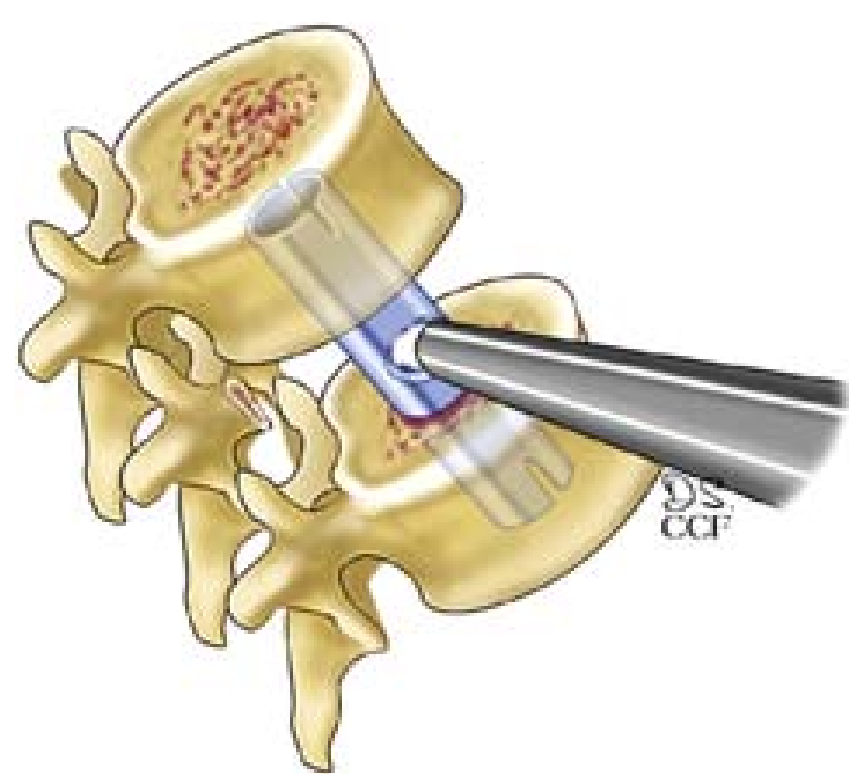

Fig. 1. Drawing showing a No. 36 French chest tube placed into the vertebrectomy defect. Methylmethacrylate is then infused through a hole created in the middle.

racic kyphosis often makes the placement of anterior instrumentation impossible in this region. Posterior instrumentation may have to be used in this case. Posteriorly, instrumentation should span the cervicothoracic junction and not end there, which may result in junctional zone kyphosis. $^{3}$

\section{Thoracic Spine}

Anteriorly, tumors may be resected either via transthoracic thoracotmoy ${ }^{15}$ or a retropleural route. ${ }^{21}$ Both allow direct visualization of the anterior thoracic spine for optimum tumor resection and for the placement of anterior instrumentation. Retropleural thoracotomy avoids the need for chest thoracostomy. The side of the operation should be based on the location of lesion(s). Often the right side is chosen when approaching lesions at T-3 and T-4, because the aortic arch limits visualization on the left side. For lesions located at T-5 through T-10, the left side is often chosen because the aorta, as opposed to the inferior vena cava, is easier to mobilize and repair if inadvertently injured.

Anterior thoracic spine lesions may also be reached via posterolateral and posterior approaches. These approaches allow anterior decompression and strut graft placement through a posterior incision, which is a procedure familiar to all spine surgeons. Because the anteriorly located lesion is not directly visualized, it may be difficult at times to achieve total tumor resection. Anterior instrumentation may not be placed using these approaches, except possibly with the LECA; therefore, posterior instrumentation is used to augment the anterior decompression. These strategies are ideal for implementation in patients whose medical conditions do not allow for thoracotomy or in whom circumferential tumors are present. A bilateral transpedicular resection has been described that allows the surgeon to perform a spondylectomy. ${ }^{1}$ Costotransversectomy and

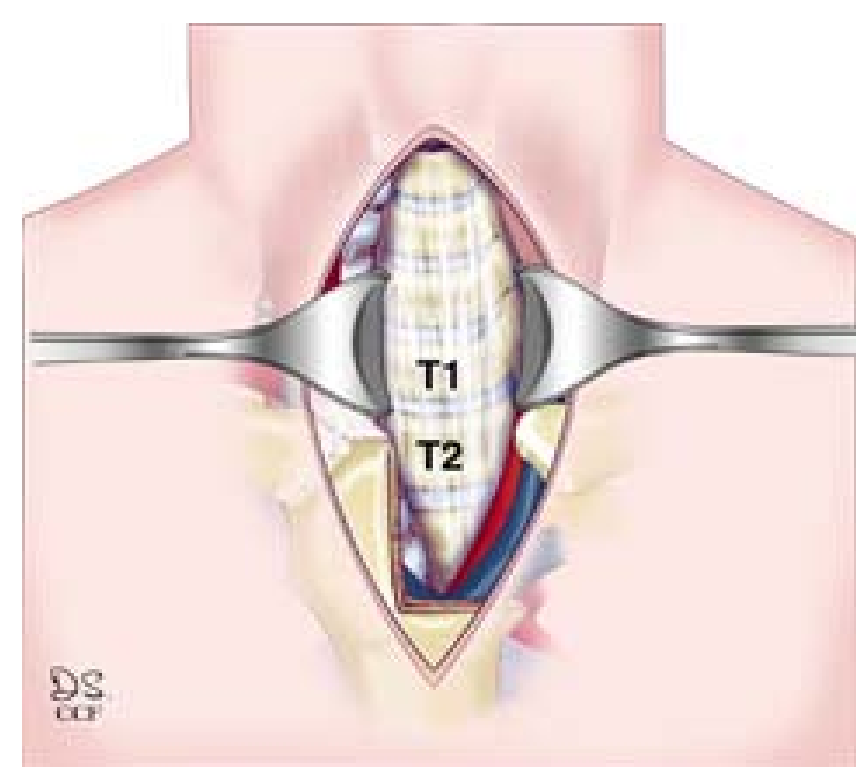

Fig. 2. Drawing. The manubrium may be resected or a window may be created in it. This may be performed using rongeurs or a high-speed drill. Care must be taken because the brachiocephalic vein lies beneath the sternal notch. This allows access to T-1, T-2, and possibly $\mathrm{T}-3$.

the LECA ${ }^{4,18}$ may also be used. They differ in the direction of erector spinae muscle retraction, the extent of VB visualization, and the working angle created (Fig. 3). The LECA is a formidable operation, but it avoids entrance into the thoracic cavity, which may be preferred. In cases of thoracolumbar lesions, the diaphragm does not require incision.

\section{Thoracolumbar Junction}

As previously mentioned, LECA may be performed in the treatment of thoracolumbar disease. Anteriorly, either a transthoracic or retropleural thoracotomy must be extended into the retroperitoneal space. Intrapleurally, the diaphragm must be incised, and the insertions to the spine must be reflected off to allow entrance into the retroperitoneal space. In a retropleural approach, the diaphragm is not incised; rather, its attachments to the spine are reflected and the retroperitoneal space is entered. Once the spine is visualized, resection and reconstruction are performed, as in the thoracic spine. In this region, consideration should be given to augmentation of an anterior decompression with posterior instrumentation. As noted in the cervicothoracic region, instrumentation should span and not end in this region. ${ }^{3}$

\section{Lumbar Spine}

Anterior pathological entities in the upper lumbar spine may be reached via a retroperitoneal approach (Fig. 4). The side of the operation should be chosen based on the location of the lesion, but the left side is often used because it is technically easier to mobilize and repair an inadvertent injury to the aorta compared with the vena cava. When a retroperitoneal approach is performed, lesions in the lower lumbar spine (L-5) are more difficult to 
resect and reconstruction is harder to perform. Because the iliac crest often hampers this approach in this region, a window may be made in the iliac crest to improve visualization. Another option would be to utilize an anterior, transperitoneal approach in this situation. This true anterior approach is useful for lesions in the lower lumbar spine. There are disadvantages, however, including violation of the peritoneum. Vertebrectomy and tumor resection allow visualization of the thecal sac. Strut grafts are easily placed and anterior instrumentation may be used. Because of the difficulty with anterior instrumentation placed at the lumbosacral junction, this region is often augmented with posterior instrumentation. Posteriorly, vertebrectomy may be performed via a bilateral transpedicular route.

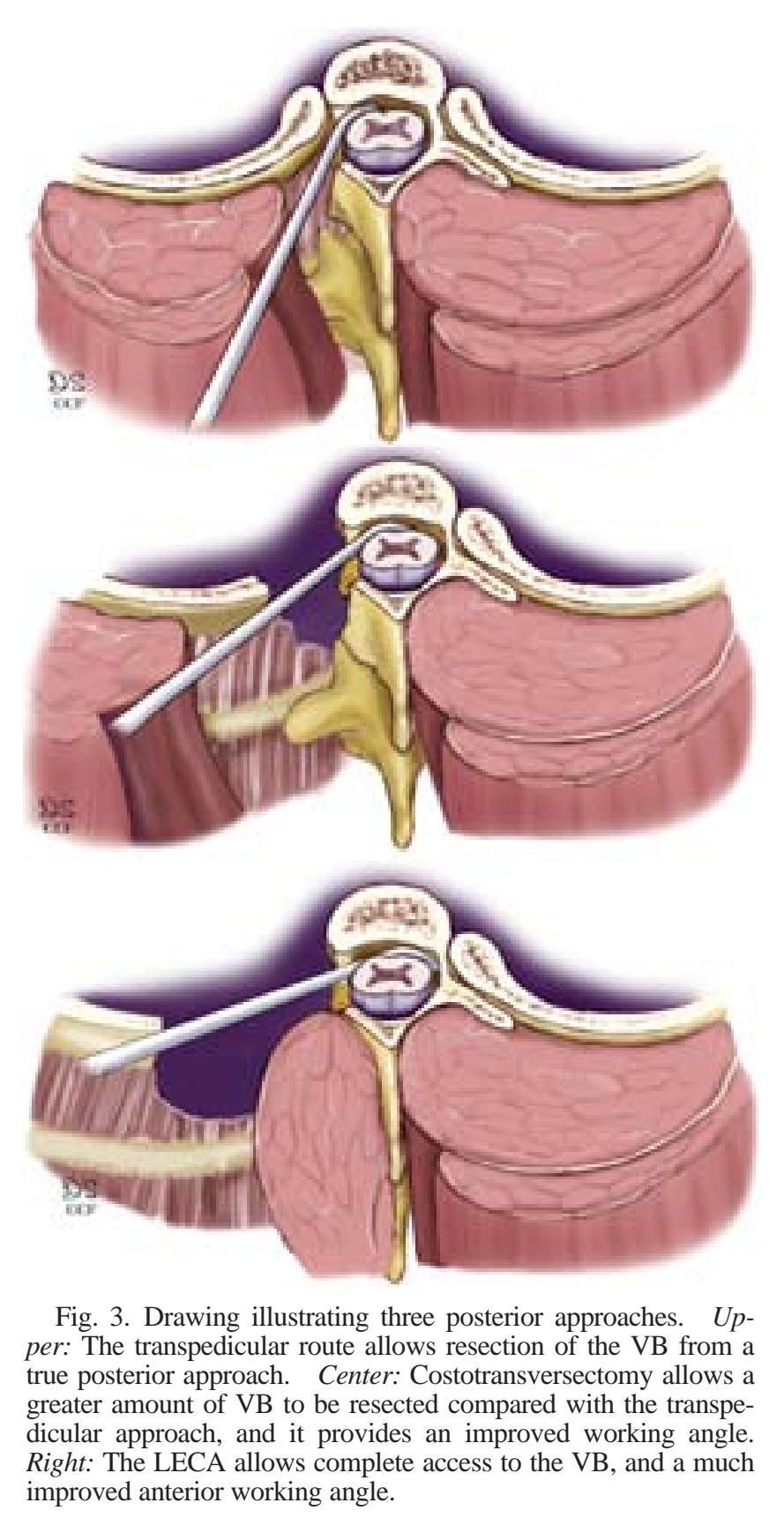

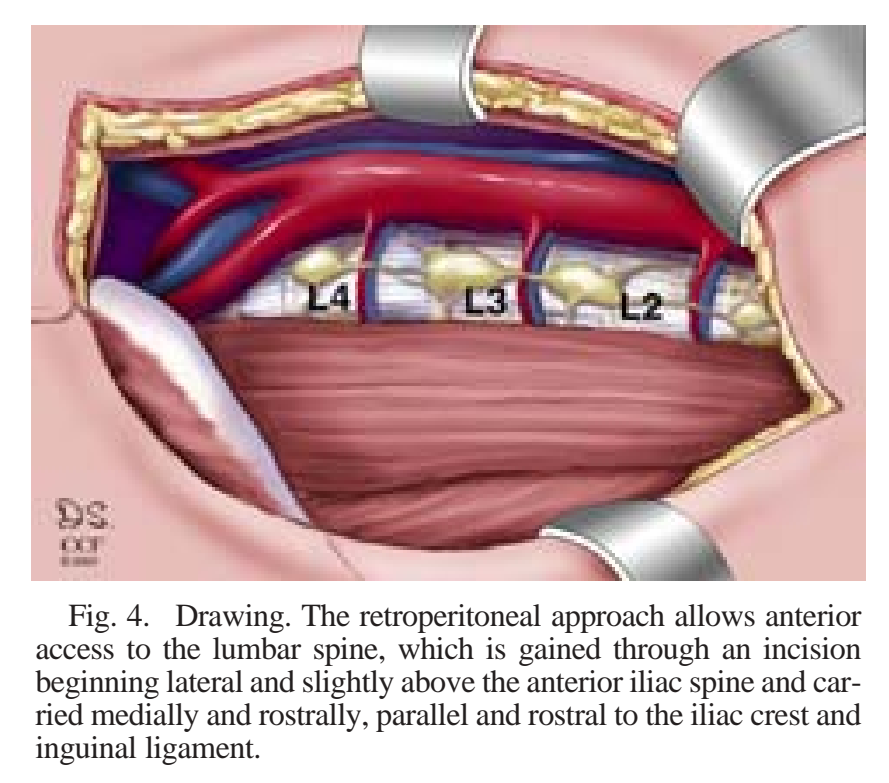

\section{CLINICAL SERIES}

\section{Anterior Surgery}

Anterior surgery is appealing because it provides the most direct route for decompression and tumor resection. Additionally, this approach allows for adequate reconstruction of the anterior vertebral column. Many studies have been performed to evaluate the results of anterior and anterolateral approaches for spinal metastasis.

Sundaresan, et al., ${ }^{29}$ have reported a prospective series of 54 patients who underwent surgery prior to radio- or chemotherapy. Patients harboring radiosensitive tumors or those in whom life expectancy was fewer than 3 months were excluded. Eighty-three percent of the patients underwent decompressive surgery via an anterior or anterolateral approach. Forty-four percent of patients were nonambulatory prior to surgery. Most were unable to walk because of weakness, whereas the second most common reason for inability to ambulate was pain. After surgery, all patients were ambulatory. Pain improved in $90 \%$. Forty-six percent of the patients were alive after 2 years, the majority of whom remained ambulatory. The overall complication rate was $20 \%$.

Harrington ${ }^{16}$ reported 77 patients with spinal instability caused by metastatic pathological fractures of one or more vertebrae. Treatment consisted of anterior decompression and stabilization with MMA augmented by knodt distraction rods positioned anteriorly. The follow-up period ranged from 31 to 146 months. Of the 62 patients with major preoperative neurological impairment, 26 experienced complete neurological recovery, 16 improved significantly, 20 remained unchanged, and one patient deteriorated. Relief of pain was obtained in 72 patients. There was one case of wound infection among the 83 anterior stabilization procedures.

Cooper, et al., ${ }^{8}$ have studied 28 patients who underwent an anterior decompression for spinal metastasis. Surgery was performed in those with spinal cord compression, spinal instability, rapidly progressive neurological deficit, failure of radiation therapy, or occult primary tumor. Sev- 
enty-five percent of the patients were ambulatory preoperatively and $88 \%$ postoperatively. Pain improved in $97 \%$ of the patients. Motor function remained the same or improved in $94 \%$ of patients. The overall complication rate was $22 \%$. The mean survival length was 10 months.

Gokaslan, et al., ${ }^{15}$ have reported on 72 patients with thoracic spine metastatic tumors. Indications for surgery included symptomatic spinal cord compression and/or intractable axial spinal pain due to spinal instability. Life expectancy exceeded 3 months. Preoperatively, 13 patients were nonambulatory. Postoperatively 10 were able to ambulate. Pain improved in $92 \%$ of patients. Thirtyfive $(76 \%)$ of 46 patients who presented with neurological impairment improved significantly following surgery. The overall complication rate was $28 \%$, and the 1 -year survival rate was $62 \%$.

Anterior and anterolateral decompression and stabilization of the spine in patients with spinal metastasis is effective. As previously mentioned, it allows a direct route for decompression of the spinal cord and tumor resection. Additionally, it allows for effective reconstruction of the anterior column. Motor function has been found to improve in 70 to $100 \%$ of patients; pain has also been found to improve in 85 to $97 \%$. $^{8,15,17,25,28-30,31}$ Wound-related complications have been reported to occur in as many as $28 \%$ of patients who have undergone postirradiation laminectomy. ${ }^{28}$ The wound-related complication rate for anterior approaches is approximately $3 \% .^{15,28}$

\section{Posterior and Posterolateral Approaches}

Although an anterior approach is optimum for tumor resection and reconstruction, these goals may also be achieved by undertaking a posterior or posterolateral approach. The operation is usually performed with uni- or bilateral facet joint or pedicle resection. This allows for anterior decompressive surgery to be performed, although often not complete, and for instrumentation to be placed posteriorly. The disadvantages are incomplete tumor resection, often suboptimum anterior graft placement, and increased incidence of wound-related complications. Anterior instrumentation can usually not be placed via a posterolateral incision. A posterior approach may be reasonable for patients harboring circumferential tumors or those who are at high risk for a more extensive anterior approach. The posterior placement of segmental instrumentation is also quite useful in cases with VB collapse at several levels and may be useful in cases requiring prophylactic stabilization. ${ }^{10}$

Cybulski, et al., ${ }^{9}$ have reported on 15 patients who underwent a modified costotransversectomy for the treatment of metastasis to the thoracic spine. Postoperative neurological improvement was seen in $83 \%$ of the patients. Sixty percent of those who were nonambulatory before surgery were able to walk postoperatively. There was one case $(7 \%)$ of wound infection requiring removal of the posterior hardware.

Akeyson and $\mathrm{McCutcheon}^{2}$ have reported on 25 patients who underwent a single-stage posterior complete spondylectomy, reconstruction, and placement of posterior instrumentation for metastatic spinal disease. Patients were chosen for this approach for several reasons: 1) their medical condition precluded making an incision on the chest or abdomen and one posteriorly; 2) risk of undue morbidity; 3) preexisting pulmonary, mediastinal, or retroperitoneal disease; 4) significant tumor located dorsal to the pedicles; and 5) involvement of two or more levels or involvement at two discontinuous levels. Surgery was performed via a bipedicular approach, with MMA reconstruction and placement of posterior instrumentation. One hundred percent of the patients with pain as their sole symptom experienced pain resolution. In $56 \%$ of those with preoperative neurological impairment, improvement was demonstrated, whereas in $39 \%$, it was unchanged and in $5 \%$ it was worse. The overall complication rate was $52 \%$, with persistent cerebrospinal fluid leakage as the most common complication. Twelve percent of the patients were alive at 1 year.

The aforementioned results demonstrate that a posterior approach may be effective in treating metastatic spinal disease. This type of approach allows the surgeon flexibility in choosing the appropriate surgical strategy based on specific patient and pathological characteristics.

\section{Combined Anterior-Posterior Approaches}

Often a combined anterior-posterior approach is deemed necessary for the treatment of patients with spinal metastasis. The indications and results of the combined anterior-posterior approach for spine tumors was studied by Sundaresan, et al. ${ }^{31}$ Indications included: 1) radiographic demonstration of three-column involvement; 2) significant vertebral instability; 3) marked kyphosis; 4) involvement of more than one VB; 5) junctional site involvement; 6) prior laminectomy; and 7) potentially solitary lesions. Forty-eight percent of their patients underwent surgery via a combined approach. Of 48 patients who were nonambulatory prior to surgery, $67 \%$ became ambulatory after surgery. Fifty-two percent of patients with severe paraparesis improved postoperatively. The overall median survival was 16 months. The complication rate was $48 \%$, with wound breakdown and infection being most common.

Of interest, Fourney, et al., ${ }^{13}$ have recently reported on a simultaneous anterior-posterior approach to the thoracic and lumbar spine for resection of tumors. They found this approach both safe and effective in cases of tumors involving both the anterior and posterior columns of the spine.

A combined approach is frequently needed, but the risk of morbidity increases. These procedures are often staged to allow the patient a resting period. Despite the increased incidence of morbidity, the majority of these complications may be managed effectively, and the patients benefit with long-term palliation and improvement in quality of life. ${ }^{31}$

\section{CONCLUSIONS}

Most spine surgeons come to treat patients with metastatic spinal disease after nonoperative management has failed. Surgery has been thought to be no better than radiotherapy based on results published in the old literature. We now have a better understanding of tumor biology, and there have been significant advances in surgical approaches and reconstructive techniques. Outcome after anterior 
decompression and stabilization is associated with pain relief, neurological improvement, and increased length of survival. Perhaps in selected patients, surgery should be chosen as first-line therapy prior to radio- or chemotherapy.

\section{References}

1. Aabo K, Walbom-Jorgensen S: Central nervous system complications by malignant lymphomas: radiation schedule and treatment results. Int J Radiat Oncol Biol Phys 12:197-202, 1986

2. Akeyson EW, McCutcheon IE: Single-stage posterior vertebrectomy and replacement combined with posterior instrumentation for spinal metastasis. J Neurosurg 85:211-220, 1996

3. Benzel EC: Biomechanics of Spine Stabilization. Rolling Meadows, IL: American Association of Neurological Surgeons, 2001

4. Benzel EC: The lateral extracavitary approach to the spine using the three-quarter prone position. J Neurosurg 71:837-841, 1989

5. Black P: Spinal metastasis: current status and recommended guidelines for management. Neurosurgery 5:726-746, 1979

6. Brihaye J, Ectors P, Lemort M, et al: The management of spinal epidural metastases. Adv Tech Stand Neurosurg 16:121-176, 1988

7. Byrne TN: Spinal cord compression from epidural metastases. N Engl J Med 327:614-619, 1992

8. Cooper PR, Errico TJ, Martin R, et al: A systematic approach to spinal reconstruction after anterior decompression for neoplastic disease of the thoracic and lumbar spine. Neurosurgery 32:1-8, 1993

9. Cybulski GR, Stone JL, Opesanmi O: Spinal cord decompression via a modified costotransversectomy approach combined with posterior instrumentation for management of metastatic neoplasms of the thoracic spine. Surg Neurol 35:280-285, 1991

10. DeWald RL, Bridwell KH, Prodromas C, et al: Reconstructive spinal surgery as palliation for metastatic malignancies of the spine. Spine 10:21-26, 1985

11. Errico TJ, Cooper PR: A new method of thoracic and lumbar body replacement for spinal tumors: technical note. Neurosurgery 32:678-681, 1993

12. Findlay GF: Adverse effects of the management of malignant spinal cord compression. J Neurol Neurosurg Psychiatry 47: 761-768, 1984

13. Fourney DR, Abi-Said D, Rhines LD, et al: Simultaneous anterior-posterior approach to the thoracic and lumbar spine for the radical resection of tumors followed by reconstruction and stabilization. J Neurosurg (Spine 2) 94:232-244, 2001

14. Gilbert RW, Kim JH, Posner JB: Epidural spinal cord compression from metastatic tumor: diagnosis and treatment. Ann Neurol 3:40-51, 1978

15. Gokaslan ZL, York JE, Walsh GL, et al: Transthoracic vertebrectomy for metastatic spinal tumors. J Neurosurg 89: 599-609, 1998

16. Harrington KD: Anterior decompression and stabilization of the spine as a treatment for vertebral collapse and spinal cord compression from metastatic malignancy. Clin Orthop 233: 177-197, 1988

17. Hosono N, Yonenobu K, Fuji T, et al: Vertebral body replacement with a ceramic prosthesis for metastatic spinal tumors. Spine 20:2454-2462, 1995
18. Larson SJ, Holst RA, Hemmy DC, et al: Lateral extracavitary approach to traumatic lesions of the thoracic and lumbar spine. J Neurosurg 45:628-637, 1976

19. Malawer MM, Delaney TF: Treatment of metastatic cancer to bone, in Devita VT, Hellman S, Rosenberg SA (eds): Cancer. Principles and Practice of Oncology, ed 4. Philadelphia: Lippincott-Raven, 1993, p 2225

20. Martenson JA Jr, Evans RG, Lie MR, et al: Treatment outcome and complications in patients treated for malignant epidural spinal cord compression (SCC). J Neurooncol 3:77-84, 1985

21. McCormick PC: The retropleural approach to the ventral thoracic and thoracolumbar spine, in Benzel EC (ed): Spine Surgery: Techniques, Complication Avoidance, and Management. Philadelphia: Churchill Livingstone, 1999, Vol 1, pp 293-304

22. McDonald DJ, Capanna R, Gherlinzoni F, et al: Influence of chemotherapy on perioperative complications in limb salvage surgery for bone tumors. Cancer 65:1509-1516, 1990

23. Nazzaro JM: Metastatic spinal lesions, in Benzel EC (ed): Spine Surgery: Techniques, Complication Avoidance, and Management. Philadelphia: Churchill Livingstone, 1999, Vol 1, pp 679-695

24. Olerud C, Jonsson H Jr, Lofberg AM, et al: Embolization of spinal metastases reduces perioperative blood loss: 21 patients operated on for renal cell carcinaoma. Acta Orthop Scand 64: 9-12, 1993

25. Perrin RG, McBroom RJ: Anterior versus posterior decompression for symptomatic spinal metastasis. Can J Neurol Sci 14: 75-80, 1987

26. Portenoy RK, Lipton RB, Foley KM: Back pain in the cancer patient: an algorithm for evaluation and management. Neurology 37:134-138, 1987

27. Saunders RL: Ventral exposures of the subaxial cervical spine, in Benzel EC (ed): Surgical Exposure of the Spine: An Extensile Approach. Illinois: American Association of Neurological Surgeons, 1995, pp 55-68

28. Siegal T, Siegal T: Surgical decompression of anterior and posterior malignant epidural tumors compressing the spinal cord: a prospective study. Neurosurgery 17:424-432, 1985

29. Sundaresan N, Digiacinto GV, Hughes JE, et al: Treatment of neoplastic spinal cord compression: results of a prospective study. Neurosurgery 29:645-650, 1991

30. Sundaresan N, Galicich JH, Lane JM, et al: Treatment of neoplastic epidural cord compression by vertebral body resection and stabilization. J Neurosurg 63:676-684, 1985

31. Sundaresan N, Steinberger AA, Moore F, et al: Indications and results of combined anterior-posterior approaches for spine tumor surgery. J Neurosurg 85:438-446, 1996

32. Walsh GL, Gokaslan ZL, McCutcheon IE, et al: Anterior approaches to the thoracic spine in patients with cancer: indications and results. Ann Thorac Surg 64:1611-1618, 1997

33. Weissman DE, Dufer D, Vogel V, et al: Corticosteroid toxicity in neuro-oncology patients. J Neurooncol 5:125-128, 1987

Manuscript received August 23, 2001.

Accepted in final form November 13, 2001.

Address reprint requests to: Edward C. Benzel, M.D., Director of Spinal Disorders, Department of Neurosurgery S-80, 9500 Euclid Avenue, Cleveland, Ohio 44195. email: benzele@ccf.org. 